\title{
SUSUSY QUANTUM MECHANICS
}

\author{
DAVID J. FERNÁNDEZ C. \\ Depto. de Física, CINVESTAV \\ A.P. 14-740, 07000 México D.F., México
}

\begin{abstract}
The exactly solvable eigenproblems in Schrödinger quantum mechanics typically involve the differential "shift operators". In the standard supersymmetric (SUSY) case, the shift operator turns out to be of first order. In this work, I discuss a technique to generate exactly solvable eigenproblems by using second order shift operators. The links between this method and SUSY are analysed. As an example, we show the existence of a twoparametric family of exactly solvable Hamiltonians, which contains the Abraham-Moses potentials as a particular case.
\end{abstract}

\section{Introduction}

The number of exactly solvable eigenproblems in non-relativistic quantum mechanics is small, and most of them can be dealt with the factorization method. This technique, introduced long ago by Schrödinger, ${ }^{1}$ was analysed in depth by Infeld and Hull, ${ }^{2}$ who made an exhaustive classification of factorizable potentials. Later on, Witten noticed the possibility of arranging the Schrödinger's Hamiltonians into isospectral pairs (supersymmetric (SUSY) partners). ${ }^{3}$ The resulting supersymmetric quantum mechanics catalysed the study of hierarchies of 'exactly solvable Hamiltonians'. An additional step was Mielnik's 'atypical' factorization ${ }^{4}$ through which the general SUSY partner for the oscillator was found; this technique was immediately applied to the hydrogen potential. ${ }^{5}$ Meanwhile, Nieto ${ }^{6}$ and Andrianov et. al. ${ }^{7}$ put the method on its natural background discovering the links between SUSY, factorization and Darboux algorithm. These developments caused the renaissance of factorization and related algebraic methods, with particular attention focused on the first order differential shift operators. ${ }^{8-16}$

As can be noticed, however, the scheme is still narrow. An obvious generalization arises when higher order differential shift operators are used to connect the Hamiltonian pair. The idea of HSUSY (higher order SUSY), recently put forward by Andrianov et. $a l .^{17-18}$ (see also ${ }^{19}$ ), incubated since 70 -tieth. ${ }^{20-21}$ In this paper we will restrict ourselves to the case when the shift operator is of second order, and we name it SUSUSY. 


\section{Second order shift operator technique}

We postulate the existence of a second order differential operator interconnecting two different Hamiltonians $H, \widetilde{H}$ :

$$
\begin{gathered}
\widetilde{H} A^{\dagger}=A^{\dagger} H, \\
H=-\frac{d^{2}}{d x^{2}}+V(x), \quad \widetilde{H}=-\frac{d^{2}}{d x^{2}}+\widetilde{V}(x), \\
A^{\dagger}=\frac{d^{2}}{d x^{2}}+\beta(x) \frac{d}{d x}+\gamma(x) .
\end{gathered}
$$

Equality (1) imposes some restrictions to the functions $\{V(x), \widetilde{V}(x), \beta(x), \gamma(x)\}$ :

$$
\widetilde{V}=V+2 \beta^{\prime}, \quad 2 V+\delta=\beta^{2}-2 \gamma-\beta^{\prime}, \quad V^{\prime \prime}+\beta V^{\prime}=2 \gamma \beta^{\prime}-\gamma^{\prime \prime},
$$

where $\delta$ is an integration constant. We shall suppose that $\beta(x)$ is given and we shall express the other functions $\{V(x), \widetilde{V}(x), \gamma(x)\}$ in terms of it. If we solve $\gamma(x)$ from second equation (4) and substitute the result in the third equation (4), we get:

$$
\frac{\beta^{\prime \prime \prime}}{2}-2 \beta^{\prime 2}+\beta^{\prime} \beta^{2}-\beta \beta^{\prime \prime}-\delta \beta^{\prime}=\beta V^{\prime}+2 \beta^{\prime} V .
$$

Multiplying by $\beta$, it can be immediately integrated, yielding:

$$
V(x)=\frac{\beta^{\prime \prime}}{2 \beta}-\left(\frac{\beta^{\prime}}{2 \beta}\right)^{2}-\beta^{\prime}+\frac{\beta^{2}}{4}+\frac{c}{\beta^{2}}-\frac{\delta}{2},
$$

where $c$ is a new integration constant. The other two unknown functions become:

$$
\tilde{V}(x)=\frac{\beta^{\prime \prime}}{2 \beta}-\left(\frac{\beta^{\prime}}{2 \beta}\right)^{2}+\beta^{\prime}+\frac{\beta^{2}}{4}+\frac{c}{\beta^{2}}-\frac{\delta}{2}, \quad \gamma(x)=-\frac{\beta^{\prime \prime}}{2 \beta}+\left(\frac{\beta^{\prime}}{2 \beta}\right)^{2}+\frac{\beta^{\prime}}{2}+\frac{\beta^{2}}{4}-\frac{c}{\beta^{2}} .
$$

Before going to the particular cases, let us notice a curious relation between the second order shift operator and Witten idea of the SUSY quantum mechanics.

\section{Second order SUSY (SUSUSY)}

According to Witten, ${ }^{3}$ SUSY arises by defining a set of operators $Q_{i}$ that commute with the (supersymmetric) Hamiltonian $H_{s}$,

$$
\left[Q_{i}, H_{s}\right]=0, \quad i=1 \cdots N
$$

and satisfy the algebra

$$
\left\{Q_{i}, Q_{j}\right\}=\delta_{i j} H_{s},
$$

where $[\cdot, \cdot]$ represents the commutator and $\{\cdot, \cdot\}$ the anticommutator. Now, with the aid of the operators $A^{\dagger}, A$ of the previous section, one can construct a case of the supersymmetric algebra (8-9) with $N=2$. 
With this aim, define the supercharges: ${ }^{17-18}$

$$
Q=\left(\begin{array}{cc}
0 & 0 \\
A & 0
\end{array}\right), \quad Q^{\dagger}=\left(\begin{array}{cc}
0 & A^{\dagger} \\
0 & 0
\end{array}\right)
$$

where $A^{\dagger}$ is given in (3) and $A$ is its adjoint. Notice that $Q^{2}=Q^{\dagger 2}=0$. Let us construct an operator, which we cannot abstain to call the SUSUSY 'Hamiltonian':

$$
H_{s s}=\left\{Q, Q^{\dagger}\right\}=\left(\begin{array}{cc}
A^{\dagger} A & 0 \\
0 & A A^{\dagger}
\end{array}\right)=\left(\begin{array}{cc}
H^{+} & 0 \\
0 & H^{-}
\end{array}\right)
$$

Using the SUSY languaje, $H^{+}=A^{\dagger} A$ and $H^{-}=A A^{\dagger}$ should be called the SUSY partners. Notice that the SUSUSY 'Hamiltonian' $H_{s s}$ commutes with the two supercharges $Q$ and $Q^{\dagger}$. The SUSY generators $Q_{1}=\left(Q^{\dagger}+Q\right) / \sqrt{2}, Q_{2}=\left(Q^{\dagger}-Q\right) / i \sqrt{2}$, and $H_{s s}$ satisfy the supersymmetric algebra (8-9).

Notice that the SUSY partners $H^{+}, H^{-}$are now the fourth order differential operators. It can be shown that $H^{+}$commutes with $\widetilde{H}$ and $H^{-}$commutes with $H$. Hence, $H^{+}$can be a certain function of $\widetilde{H}$ and $H^{-}$a function of $H$. Indeed:

$$
H^{+}=\left(\widetilde{H}+\frac{\delta}{2}\right)^{2}-c, \quad H^{-}=\left(H+\frac{\delta}{2}\right)^{2}-c .
$$

A physical Hamiltonian $H_{s}$ can be defined (compare with the recent ideas of ${ }^{17-18}$ ),

$$
H_{s}=\left(\begin{array}{cc}
\widetilde{H} & 0 \\
0 & H
\end{array}\right)
$$

and the SUSUSY 'Hamiltonian' $H_{s s}$ is related to $H_{s}$ by means of:

$$
H_{s s}=\left(H_{s}+\frac{\delta}{2}\right)^{2}-c
$$

Thus, the SUSUSY 'Hamiltonian' $H_{s s}$ is a quadratic form of a physical Hamiltonian $H_{s}$. The diagonal elements of $H_{s}$ are the two Hamiltonians $H, \widetilde{H}$ of the previous section, which are related by the second order differential operators $A, A^{\dagger}$ (compare ${ }^{17-18}$ ). 


\section{Example: the SUSUSY oscillator}

We shall now look for the SUSUSY analogue of the oscillator Hamiltonian

$$
H=-\frac{d^{2}}{d x^{2}}+x^{2}
$$

We will try to show the existence of a 2-parametric family of potentials isospectral to $V(x)=x^{2}$. This has to do with the general solution $\beta(x)$ of equation (6). This solution of course should include the ladder operator $A^{\dagger}=\left(a^{\dagger}\right)^{2}$, where $a^{\dagger}=-d / d x+x$ is the standard ladder operator of the harmonic oscillator. This means that for $V(x)=x^{2}$ and $\beta_{p}(x)=-2 x$, equation (6) should become an identity, which fixes the constants to $c=1, \delta=4$. Substituting these results again in (6) and multiplying by $2 \beta^{2}$, we get:

$$
\beta \beta^{\prime \prime}-\frac{\beta^{\prime 2}}{2}-2 \beta^{2} \beta^{\prime}+\frac{\beta^{4}}{2}-4 \beta^{2}-2 x^{2} \beta^{2}+2=0 .
$$

Let us notice the existence of an explicit solution more general than $\beta_{p}=-2 x$. It arises after multiplying the standard raising operator $a^{\dagger}$ by the operator $b^{\dagger}$ of atypical factorizations, ${ }^{4}$ i.e., $A^{\dagger}=b^{\dagger} a^{\dagger}$, leading to:

$$
\beta_{p}(x)=-2 x-\frac{e^{-x^{2}}}{\lambda+\int_{0}^{x} e^{-y^{2}} d y}, \quad|\lambda|>\frac{\sqrt{\pi}}{2} .
$$

The general solution of (16), which depends on two constants, should reduce itself to (17) as one of them takes a particular value (or one of them becomes a function of the other one).

Here, I would like to present some partial numeric results obtained when (16) is integrated to provide $\beta(x)$ which arises as continuous deformations of the particular solutions (17). With this aim we choose the initial point $\left(\beta(0), \beta^{\prime}(0)\right)$ on the Poincaré plane close to $\left(\beta_{p}(0), \beta_{p}^{\prime}(0)\right)$ and use then a standard numeric integration package ${ }^{\mathrm{a}}$ to find $\beta(x)$ and to look for singularities in the corresponding SUSUSY potential $\tilde{V}(x)=x^{2}+2 \beta^{\prime}(x)$. If there is no singularity, we increase slightly $\beta^{\prime}(0)$ maintaining $\beta(0)$ fixed, and repeat the integration until finding the upper threshold of $\beta^{\prime}(0)$ : above this threshold a singularity arises while below it disappears. A similar procedure is used to find the low threshold. After that, we make a small change of $\beta(0)$ along $\left(\beta_{p}(0), \beta_{p}^{\prime}(0)\right)$ and start again the whole process. In this way we can split the $\beta \beta^{\prime}$-plane into the region where the SUSUSY potential $\tilde{V}(x)$ is free of singularities and the rest. Notice that the points $\left(\beta_{p}(0), \beta_{p}^{\prime}(0)\right)$ provide a curve on $\beta \beta^{\prime}$-plane:

$$
\beta_{p}^{\prime}(0)=-2+\beta_{p}^{2}(0), \quad\left|\beta_{p}(0)\right|<\frac{2}{\sqrt{\pi}} .
$$

aWe have employed the routine 'NDSolve' of 'Mathematica' 
Departing from (18) we made the classification on figure 1.

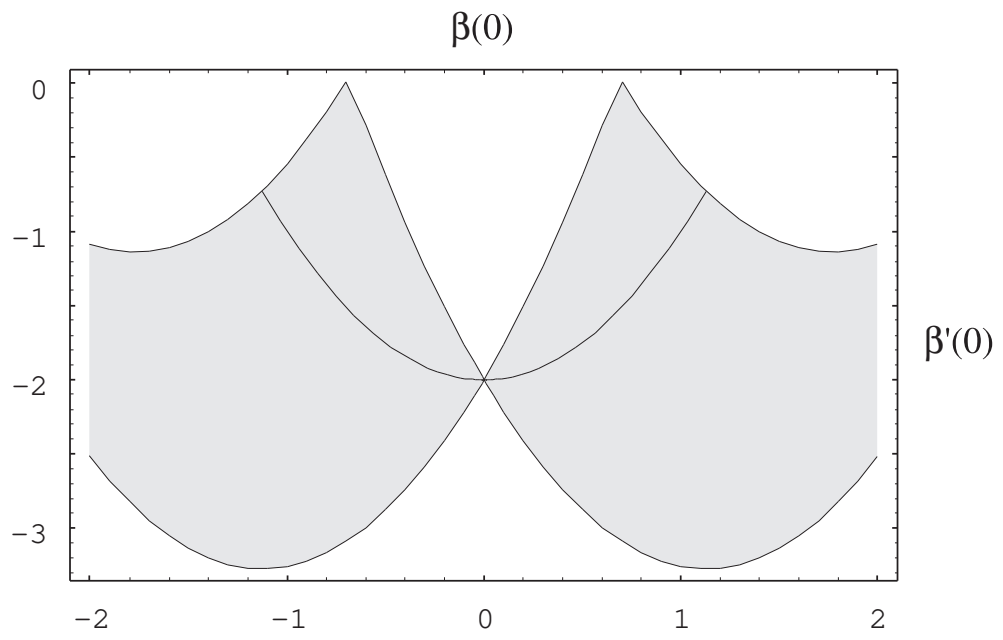

Fig. 1. Classification of the $\beta \beta^{\prime}$-plane into regions where the SUSUSY potential $\widetilde{V}(x)$ has no singularities (the shadowed regions) and those with singularity (the white regions).

As we can see, there is a non-trivial region, the shadowed one, where the SUSUSY potential $\widetilde{V}(x)$ has no singularity; it comprises the curve in (18). For the purposes of this paper, to show that the general family of SUSUSY oscillator potentials is two parametric, our calculation brings already some information.

The SUSUSY potentials $\widetilde{V}(x)$ for various values of the pair $\left(\beta(0), \beta^{\prime}(0)\right)$ lying at the shadowed region (no singularity, we have fixed $\beta(0)=-0.7$ ) are shown in figure $2^{\mathrm{b}}$. This family is richer than the Abraham-Moses (AM) SUSY potentials: ${ }^{4,22}$

$$
V_{\lambda}(x)=x^{2}-2 \frac{d}{d x}\left(\frac{e^{-x^{2}}}{\lambda+\int_{0}^{x} e^{-y^{2}} d y}\right)
$$

This is so because $\widetilde{V}(x)$ is essentially two-parametric while (19) is just one-parametric. Indeed, the $V_{\lambda}(x)$ of (19) can be numerically reconstructed by solving (16) with the points of (18) as the initial conditions. The SUSUSY family obtained by this procedure coincides with a plot of the analytic results (19).

bIndeed, $\widetilde{V}(x)$ is displaced with respect to $V(x)=x^{2}$ a quantity $\delta E=-4$. This can be seen after realizing that $\widetilde{V}(x)+4 \rightarrow x^{2}$ when $\left(\beta(0), \beta^{\prime}(0)\right) \rightarrow(0,-2)$. Hence, we decided to represent on the vertical axis of figure 2 the potentials $\widetilde{V}(x)+4$ rather that $\widetilde{V}(x)$. 


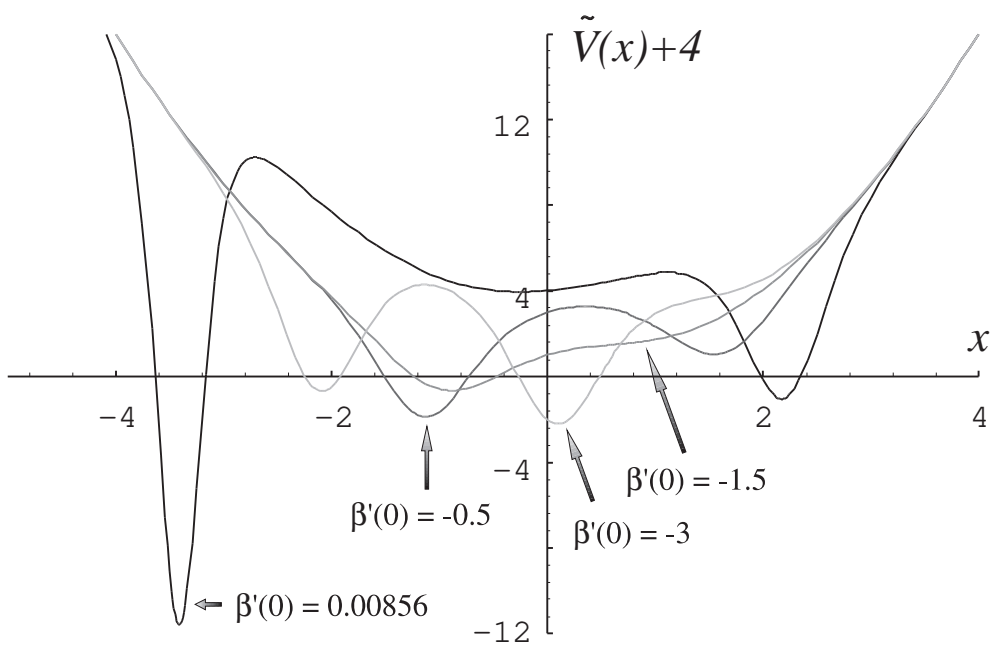

Fig. 2. The SUSUSY potentials $\widetilde{V}(x)+4$ for some values of $\beta^{\prime}(0)$ and $\beta(0)=-0.7$. All pairs $\left(\beta(0), \beta^{\prime}(0)\right)$ lie in the region where there is no singularity for $\widetilde{V}(x)$.

Interesting that the SUSUSY family $\widetilde{V}(x)$ embraces some cases of the widely discussed double well potentials (DWP). The dynamics of a system in these potentials is of some relevance, because it illustrates the differences between the classical and quantum regimes. In particular, it clearly shows one of the most intriguing quantum effects, the tunneling of the system from one well to the other as a result of the evolution. In most of the situations where a DWP is a SUSY pair of the oscillator potential, the DWP spectrum has one level more below the ground state energy of the oscillator. Moreover, it is usually symmetric with respect to $x=0$ (see e.g. ${ }^{9,23}$ ). For our SUSUSY DWP, apparently, it is unneccessary to add any level below the ground state energy of the oscillator to generate the double well: the spectra of $\widetilde{V}(x)+4$ and $V(x)=x^{2}$ are equal. ${ }^{\text {c }}$ The price to pay is that $\widetilde{V}(x)$ and $V(x)=x^{2}$ are not precisely the SUSY partners, as shown in section 3. Moreover, although $\widetilde{V}(x)$ is a double well, it turns out that it is not symmetric with respect to any point $x=x_{0}$. We hope that the SUSUSY treatment here presented can be implemented to other physically interesting potentials.

\section{Acknowledgements}

The support of CONACYT is acknowledged.

${ }^{\mathrm{c}}$ This is at the moment a hypothesis supported by our numerical plots of $\beta(x)$ and $\widetilde{V}(x)$. (The continuity argument might be important.) 


\section{References}

1. E. Schrödinger, Proc. Roy. Irish Acad. A 46, 183 (1940); A 47, 53 (1941).

2. L. Infeld and T.E. Hull, Rev. Mod. Phys. 23, 21 (1951).

3. E. Witten, Nucl. Phys. B188, 513 (1981); B202, 253 (1982).

4. B. Mielnik, J. Math. Phys. 25, 3387 (1984).

5. D.J. Fernández C., Lett. Math. Phys. 8, 337 (1984).

6. M.M. Nieto, Phys. Lett. 145B, 208 (1984).

7. A.A. Andrianov, N.B. Borisov and M.V. Ioffe, Phys. Lett. 105A, 19 (1984).

8. A. Frank and K.B. Wolf, J. Math. Phys. 26, 973 (1985).

9. C.V. Sukumar, J. Phys. A 18, L57 (1985); A 18, 2917 (1985); A 18, 2937 (1985).

10. Z. Dongpei, J. Phys. A 20, 4331 (1987).

11. N.A. Alves and E. Drigo-Filho, J. Phys. A 21, 3215 (1988); E. Drigo-Filho, J. Phys. A 21, L1025 (1988).

12. G. Levai, J. Phys. A 22, 689 (1989).

13. A. Stahlhofen and K. Bleuler, Nuov. Cim. 104B, 447 (1989).

14. A. Arai, Lett. Math. Phys. 19, 217 (1990).

15. O.L. de Lange, Am. J. Phys. 59, 151 (1991); O.L. de Lange and R.E. Raab, Operator methods in quantum mechanics, Clarendon Press, Oxford (1991).

16. D.J. Fernández C., J. Negro and M.A. del Olmo, Ann. Phys. 252 (1996).

17. A.A. Andrianov, M.V. Ioffe and V. Spiridonov, Phys. Lett. 174A, 273 (1993).

18. A.A. Andrianov, M.V. Ioffe, F. Cannata and J.-P. Dedonder, Int. J. Mod. Phys. A 10, 2683 (1995).

19. V.M. Eleonsky and V.G. Korolev, J. Phys. A 28, 4973 (1995).

20. M. Moshinsky, J. Patera and P. Winternitz, J. Math. Phys. 16, 82 (1975); R.W. Carrol, Transmutation and Operator Differential Equations, North-Holland Mathematics Studies 37, The Netherlands (1979).

21. D.J. Fernández C., M.Sc. thesis, CINVESTAV, 1984 (in spanish).

22. P.B. Abraham and H.E. Moses, Phys. Rev. A 22, 1333 (1980).

23. B.N. Zakhariev, N.A. Kostov and E.B. Plekhanov, Sov. J. Part. Nucl. 21, 384 (1990); V.M. Chabanov and B.N. Zakhariev, Phys. Rev. A 49, 3159 (1994). 